\title{
The effect of plasma triangularity on turbulent transport: modeling TCV experiments by linear and non-linear gyrokinetic simulations
}

\author{
A Marinoni ${ }^{1}$, S Brunner ${ }^{1}$, Y Camenen ${ }^{2}$, S Coda ${ }^{1}$, J P Graves ${ }^{1}$, \\ X Lapillonne ${ }^{1}$, A Pochelon ${ }^{1}$, O Sauter $^{1}$, L Villard $^{1}$ and the TCV Team \\ ${ }^{1}$ Ecole Polytechnique Fédérale de Lausanne (EPFL), Centre de Recherches en Physique des \\ Plasmas, Association Euratom-Confédération Suisse, CH-1015 Lausanne, Switzerland \\ 2 Centre for Fusion, Space and Astrophysics, Department of Physics, University of Warwick, \\ Coventry CV4 7AL, UK \\ E-mail: alessandro.marinoni@epfl.ch
}

Received 2 September 2008, in final form 17 February 2009

Published 31 March 2009

Online at stacks.iop.org/PPCF/51/055016

\begin{abstract}
The effect of plasma shape on confinement has been experimentally explored in the TCV tokamak revealing that the core electron heat transport is significantly reduced by a negative triangularity configuration, which could indicate a (partial) stabilization of the microinstabilities at play in a conventional positive triangularity configuration.

This work is a theoretical investigation of the effect exerted by triangularity on plasma turbulence. In particular, it compares the TCV experimental results with non-linear local gyrokinetic simulations performed on the basis of actual MHD equilibrium reconstructions.

In both the linear and non-linear phases, negative triangularity is found to have a stabilizing influence on ion-scale instabilities, specifically on the so-called trapped electron mode (TEM) which is the dominant instability in the conditions of the TCV experiments considered; more specifically, the variation of the heat flux with triangularity calculated by the non-linear simulations is in fair agreement with the experimental results.

The resulting stabilization is a result of a rather complex modification of the toroidal precessional drift of trapped particles exerted by negative triangularity.

(Some figures in this article are in colour only in the electronic version)
\end{abstract}

\section{Introduction and overview of TCV experiments}

Plasma shape has been theoretically and experimentally recognized as a major player in plasma performance through its effect on both MHD and microturbulence. A fusion relevant tokamak 
needs to operate at high pressure and possibly at high bootstrap current to maximize the reactor efficiency by means of a high fusion rate and, in the case of advanced-tokamak scenarios, a low externally driven current. However there are limits to the density, pressure and current which a plasma can withstand and above which MHD instabilities occur, destroying the desired configuration. The influence of plasma shape on MHD stability was theoretically studied in $[2,3]$ and has been experimentally observed in the TCV, DIII-D and JET tokamaks in various scenarios [3-6].

The improvement in MHD stability performance with shaping in an elongated plasma is due to the increased maximum current (at fixed safety factor) carried by an elongated plasma, which in turn increases the maximum achievable beta according to the Troyon scaling [7].

Concerning the effect of shaping on microturbulence, a few linear gyrokinetic (GK) studies have been performed, showing in general a stabilizing effect of elongation on ion temperature gradient (ITG) modes [8,9] and trapped electron modes (TEMs) [10]. More recently the first non-linear GK attempts to characterize the influence of plasma shape on ITG turbulence, with the local GS2 code [11], have confirmed the stabilizing effect of elongation [12]. When performing elongation studies, it is important to consider the effect of the total current because, as non-linear results obtained with the ORB5 code [13] have revealed, at fixed shape and at fixed pressure and current profiles a larger total current reduces ITG non-linear transport according to the scaling $\chi_{i} \propto 1 / I_{\text {tot }}[14]$. This results from the consequent rescaling of the safety factor which GAMs and zonal flows depend on. Latest results from the GYRO code [15] indicate that elongation is beneficial in regard to a mixed ITG/TEM turbulence.

All the analyses performed so far either focused on elongation alone or found that the effect of triangularity is negligible in comparison. In contrast, in this paper we focus almost exclusively, both linearly and non-linearly, on modeling the effect of triangularity on confinement as observed in TCV [1].

The TCV tokamak, Tokamak á Configuration Variable [16], was specifically designed and built to explore the influence of shape on the plasma properties. Indeed, it can operate with edge elongation between 1 and 2.8 and with edge triangularity between 1 and -0.7 . Dedicated experimental campaigns were devoted to studying the influence of plasma shape on energy confinement in L-mode; this choice was dictated primarily by the need to minimize the influence of the magnetic geometry on plasma edge stability and focus on core transport studies. Initially, ohmic plasmas were considered with line averaged densities ranging from 5 to $9 \times 10^{19} \mathrm{~m}^{-3}$ and plasma elongation, $\kappa$, between 1 and 2.7 and triangularity, $\delta$, between -0.3 and 0.55 . The energy confinement time, $\tau_{E}$, increased considerably with elongation but was independent of triangularity [17-19]. This behavior was explained by a steepening of the average temperature gradient due to flux surface compression, whereas no dependence of the underlying electron diffusivity on plasma shape was observed. At a later stage, L-mode centrally EC-heated plasmas were studied at a lower line averaged density $1.8 \times 10^{19} \mathrm{~m}^{-3}$, intermediate elongation $\kappa=1.5$ and triangularity between -0.65 and 0.55 , revealing a strong dependence of the electron energy confinement time on triangularity, which could be cast in the form $(1+\delta)^{-0.35}$ [20], and could not be explained by the flux surface compression effect mentioned before. These results motivated a detailed triangularity scan of EC-heated plasmas. Elongation was kept fixed, at $\kappa=1.6$, because any change in it introduces major changes in plasma parameters such as current and volume which seriously complicate the analysis. The electron heat transport is calculated via power balance analysis, taking into account EC power deposition, ohmic power and electron-ion equipartition. Interested readers are referred to $[1,21]$ for further details. The main result of the experiments was the stabilizing role of negative triangularity: in particular, the same electron temperature and density profiles were achieved for plasmas with triangularity of the last closed flux surface (LCFS) equal to 0.4 

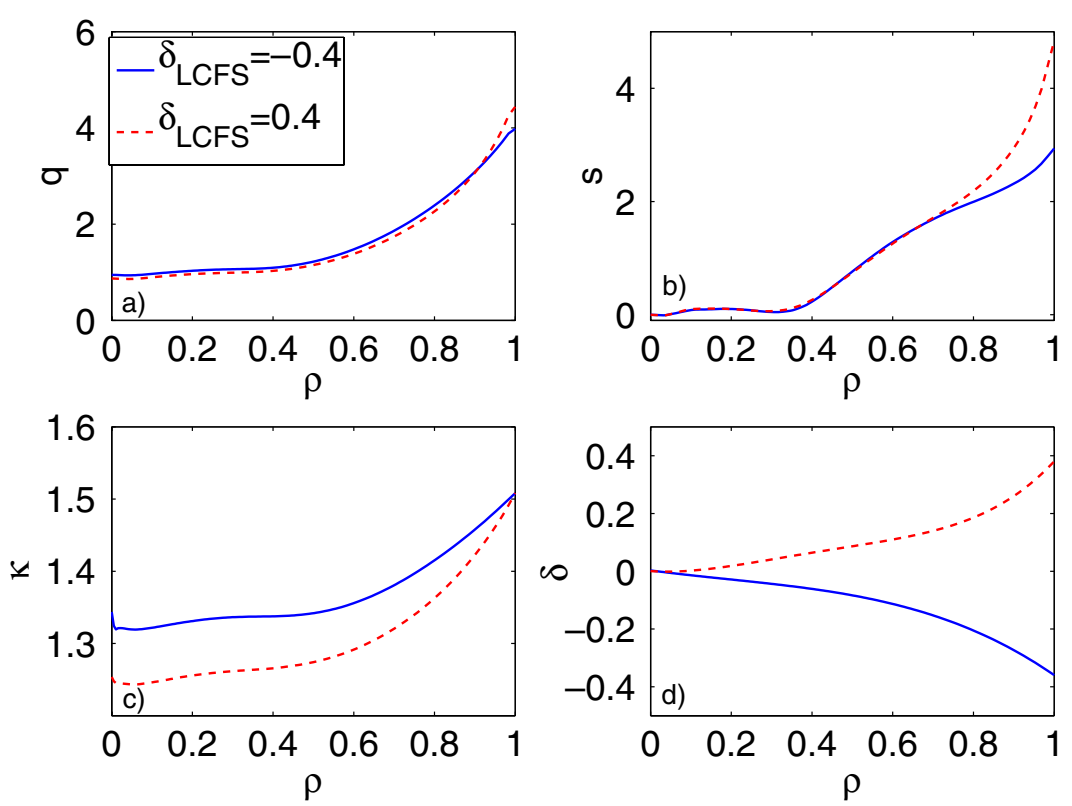

Figure 1. (Color online.) Overview of the equilibrium of TCV shots 28008 and 28014. Radial profiles of safety factor $(a)$, magnetic shear $(b)$, elongation $(c)$ and triangularity $(d)$. The $q$ profiles in $(a)$ have been more accurately calculated than in [1].

and -0.4 , respectively, injecting in the latter case half as much power as in the former. This implies that flipping the edge triangularity from 0.4 to -0.4 essentially halves the electron heat transport. Conversely, injecting the same amount of EC power resulted in considerably higher temperature in the $\delta<0$ case with respect to the $\delta>0$ one. In the rest of this paper we will focus on two TCV shots (shots 28014 and 28008) having the same electron density and temperature profiles but two different edge triangularity values, being equal to 0.4 and -0.4 , respectively. These two shots were analyzed in [1], whose figures 2 and 1 in this paper provide an overview. They are characterized by having the same kinetic profiles within the errorbars except for the ion temperatures, which were about $15 \%$ higher in the center for the negative $\delta$ case and approximately the same at $\rho=0.7$; however in the simulations we will neglect this effect which, on the basis of linear simulations, is expected to be small. Note in particular that $T_{\mathrm{e}} / T_{\mathrm{i}}$ is larger than two in these discharges.

These TCV discharges motivated this study which investigates their microstability, thus assessing the direct influence of plasma shape on electron transport coefficients. It is in general difficult to quantitatively compare GK heat diffusivities with actual experimental values, due, on the one hand, to all models being incomplete representations of physical systems and, on the other hand, to error bars in kinetic and equilibrium profiles; especially when the parameter being studied is a high order moment of plasma shape, such as triangularity, which is not expected to have a large impact on plasma performance. Nevertheless, in view of the striking experimental results of [1], it is our goal to put this expectation to the test by exploring all variations due to triangularity that clearly exceed the experimental error bars.

The paper is organized as follows: in section 2 and appendix we describe the numerical tools and the simulation methodology; in section 3 we present linear simulations aimed at a coarse investigation of shape effects on transport and particle drifts; section 4 presents the core 

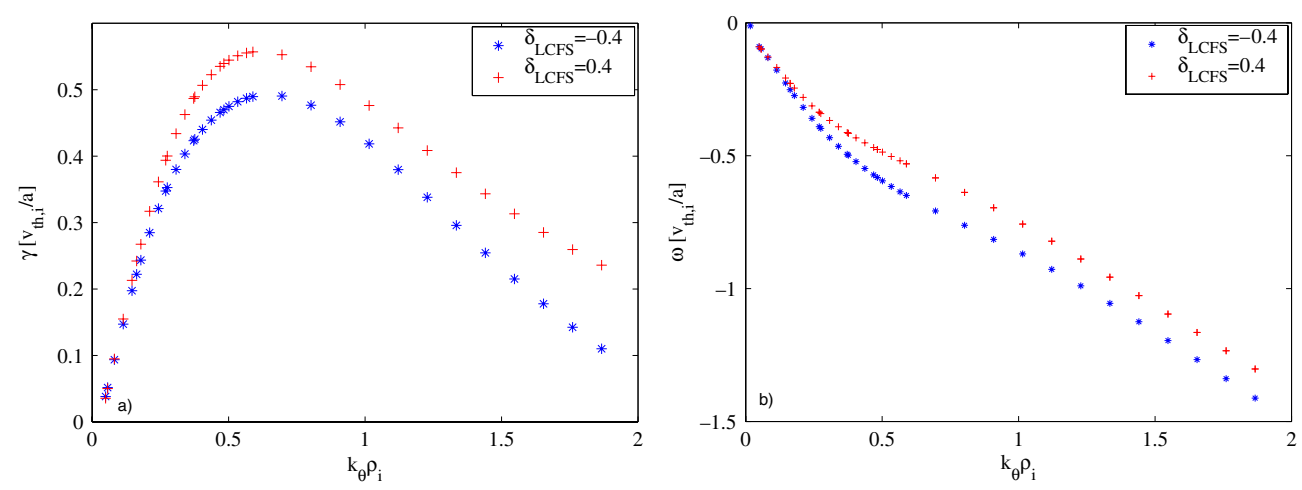

Figure 2. (Color online.) TEM dispersion relations from linear GS2 simulations for TCV shots 28014 and 28008 (growth rate on the left, real frequency on the right). The negative $\delta$ case is characterized by lower growth rates than the positive one.

non-linear studies, while section 5 discusses the interpretation of the results; conclusions are offered at the end.

\section{Methodology of numerical simulations}

The simulations have been performed with the flux tube code GS2 [11], which solves the GK Vlasov-Maxwell system of equations as an initial value problem. The code employs a ballooning representation for the linear terms, solved implicitly, and an explicit flux tube domain treatment for the non-linear terms. The code can handle different ion species and collision operators (a pitch angle diffusion operator has been used for this work) and is fully electromagnetic, even though the simulations performed here are in the electrostatic limit owing to the low beta values in the experiments considered $\left(2 \mu_{0}\langle p\rangle / B_{0}^{2} \simeq 10^{-3}\right)$. The simulations are performed with three kinetic species (electrons, deuterium and carbon as impurity), 16 to 32 energy grid points, 20 to 40 circulating-particle pitch angles and 16 to 32 trapped particle pitch angles. Carbon was retained as an impurity due to the high $Z_{\text {eff }}$ which, being larger than 3.5 , leads to a $\mathrm{C}$ concentration equal to $20 \%$ of that of deuterium. We performed separate convergence studies for positive and negative triangularities, of which the former case is slightly more demanding, and they indicate that at least 11 poloidal modes and 70 radial modes are necessary to attain an accuracy of about $8 \%$ on the saturated heat flux; this value will be used in the following as an upper-limit estimation of the error bar associated with every non-linear simulation. All the non-linear simulations discussed in this paper have been performed with 15 poloidal modes and 85 radial modes in a simulation domain which, at $\theta=0$, is $132.9 \rho_{\mathrm{i}}$ wide in the $\theta$ direction and $207.3 \rho_{\mathrm{i}}$ wide in the radial direction, resulting in turbulent modes evolved in the following range $0 \leqslant k_{\theta} \rho_{\mathrm{i}} \leqslant 0.67,\left|k_{x} \rho_{\mathrm{i}}\right| \leqslant 1.27$ and $\Delta k_{x} \rho_{\mathrm{i}}=0.03, \Delta k_{\theta} \rho_{\mathrm{i}}=0.05$; here $\rho_{\mathrm{i}}$ is the ion Larmor radius and $\theta$ is the poloidal angle along the field line. The grid limits have been chosen after exploratory non-linear runs were performed over a broader range. In particular, the upper limit $k_{\theta} \rho_{\mathrm{i}}<0.67$, which is close to the most unstable mode as seen in figure $2(a)$, is in fact well above the wave number generating the maximum heat flux, which is $k_{\theta} \rho_{\mathrm{i}} \simeq 0.15$; at $k_{\theta} \rho_{\mathrm{i}}=0.67$ the heat flux drops to $7 \%$ of the maximum (see also figure $3(b)$ ). A typical run requires about $3 \times 10^{4}$ dynamically adjusted time steps. An initial equilibrium reconstruction to determine the plasma boundary was followed by a simulation with the PRETOR [22] transport code to derive the steady-state current profile, which was then provided in input 

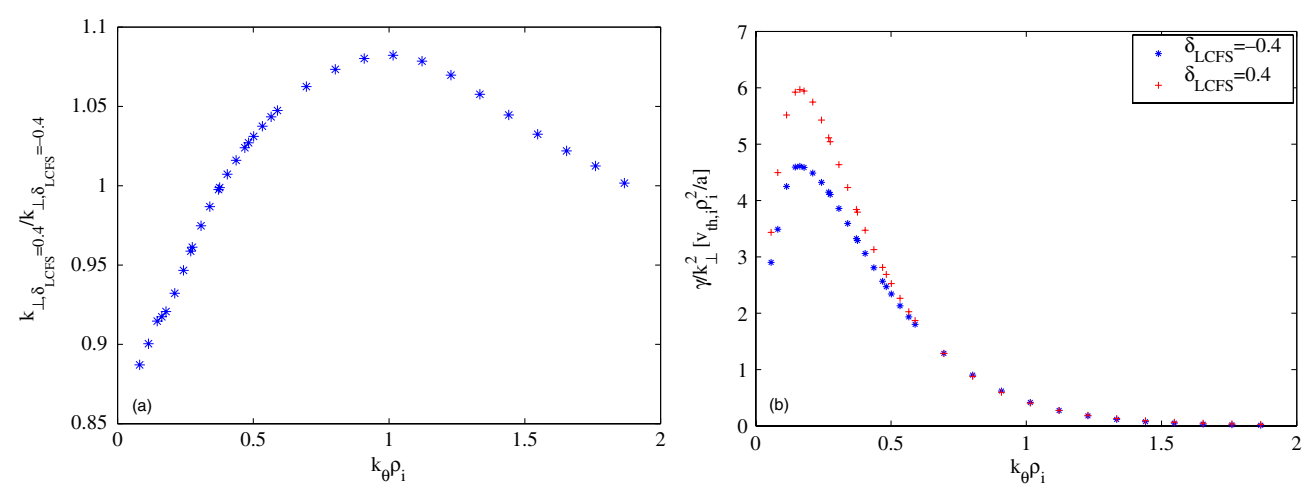

Figure 3. (Color online.) (a) Ratio of $k_{\perp}$ for TCV shots 28008 and 28014, estimated from equation (1), as a function of $k_{\theta}$. (b) Mixing-length estimate via equation (17) of the electron heat flux calculated by GS2 at $\rho=0.7$ for TCV shots 28008 and 28014.

to the CHEASE [23] equilibrium code to calculate the complete equilibria directly read by GS2. This method ensures a correct evaluation of the magnetic geometry. Alternatively, an analytical description, such as the Miller parametrization [24], could be used. However, in this case it would be imperative to choose the parameters such that they are all consistent with the equilibrium they refer to. Let us illustrate this point in the case of a triangularity scan: by solely changing the value of the triangularity on a given flux surface one does not obtain the actual experimental equilibrium relative to the desired triangularity because, in reality, triangularity is correlated with its radial derivative and with the surface's Shafranov shift, which have to be changed as well. To correctly evaluate the additional geometrical terms needed in the analytical parametrization, an actual equilibrium reconstruction is therefore required. The method employed directly reads the output of an MHD equilibrium code (CHEASE) thus automatically providing the correct geometrical information to GS2.

To isolate just the effect of plasma shape, the GS2 simulations have been performed by keeping fixed temperature, density and effective charge profiles when comparing different triangularities; the actual experimental profiles are indeed identical within the error bars.

\section{Linear simulations}

Before performing non-linear analysis, linear simulations are useful to understand the basic properties of the instabilities under consideration and to explore all underlying features of the experimental regime, in particular to uncover possible hidden variables that may affect turbulence independently of triangularity. All the runs described in this section have been performed with three kinetic species: electrons, deuterium, and carbon as impurity, the radial wave number has been chosen to be equal to zero and with the actual reconstructed equilibrium located at $\rho=0.7$, where $\rho$ is defined as the squared root of the normalized volume. The choice of this radial location corresponds to a compromise between the interest of simulating core turbulence and the fact that shape effects are more visible at the plasma edge. Indeed shape and toroidicity decrease when going from the edge to the magnetic axis.

Since the real frequencies of all unstable modes evolved are in the electron diamagnetic direction (negative sign in our convention) and since the majority of the heat flux is carried by the trapped electrons, we can conclude that the core of these TCV plasmas is dominated by TEM turbulence. To strengthen our confidence in this claim and to assess the impact of 
Table 1. Linear scan in experimental profiles showing the maximum variation in $\omega$ and $\gamma$, calculated over the linear spectrum of figure 2 , obtained by varying the equilibrium profile values within the experimental error bars.

\begin{tabular}{llll}
\hline Case & Variation & $\max \Delta \omega$ & $\max \Delta \gamma$ \\
\hline$R / L_{T_{\mathrm{e}}}$ & $-10 \%$ & $-4 \%$ & $-5 \%$ \\
$R / L_{n_{x}}$ & $-25 \%$ & $-10 \%$ & $-15 \%$ \\
$T_{\mathrm{e}} / T_{\mathrm{i}}$ & $-25 \%$ & $-16 \%$ & $-10 \%$ \\
$T_{\mathrm{e}} / T_{\mathrm{i}}$ & $+25 \%$ & $+22 \%$ & $-13 \%$ \\
$R / L T_{\mathrm{i}}$ & $+10 \%$ & $+3 \%$ & $+2.4 \%$ \\
\hline
\end{tabular}

experimental uncertainties, we performed additional simulations varying the electron and ion temperature profiles as well as the density profiles to see how far from a possible regime transition these data are; the results are summarized in table 1 and do not show any change of behavior. The $T_{\mathrm{e}} / T_{\mathrm{i}}$ ratio has been changed by $25 \%$ even though its experimental uncertainty is lower.

The calculated spectra for TCV shots 28014 and 28008 are depicted in figures 2 and 3 and show two important features. First, the growth rate of the most unstable mode for each poloidal wave vector is lower in the negative $\delta$ case compared with the positive one. Second, the values of the reconstructed field line averaged $k_{\perp}$ are, for low toroidal mode number, higher in the negative $\delta$ case with respect to the positive one, while the opposite holds for high mode numbers. The average $k_{\perp}$ has been calculated as follows [27]:

$$
\left\langle k_{\perp}^{2}\right\rangle=\frac{\int_{\theta_{\min }}^{\theta_{\max }} \mathrm{d} \theta k_{\perp}^{2}(\theta)|\tilde{\phi}(\theta)|^{2}}{\int_{\theta_{\min }}^{\theta_{\max }} \mathrm{d} \theta|\tilde{\phi}(\theta)|^{2}},
$$

where $\tilde{\phi}$ is the fluctuating electrostatic potential, $k_{\perp}$ is defined as in equation (17) and $\theta_{\min , \max }$ specify the flux tube length used in the simulation. This formula weights the perpendicular wave vector with the energy of the fluctuations, therefore in the case of strongly ballooning modes it enhances the contribution from the low field side of the plasma. When only the modes at $\theta_{0}=0$ are considered, which are usually the most unstable, it can be seen that in the high aspect ratio, zero $\beta$, circular limit equation (1) reduces to

$$
\left\langle k_{\perp}^{2}\right\rangle=k_{\theta}^{2}\left[1+s^{2} \frac{\int_{\theta_{\min }}^{\theta_{\max }} \mathrm{d} \theta \theta^{2}|\tilde{\phi}(\theta)|^{2}}{\int_{\theta_{\min }}^{\theta_{\max }} \mathrm{d} \theta|\tilde{\phi}(\theta)|^{2}}\right]=k_{\theta}^{2}\left(1+s^{2}\left\langle\theta^{2}\right\rangle\right),
$$

in agreement with [27]. The consequence of these two effects is, in a mixing-length picture, a double stabilization at low mode numbers, where most of the transport occurs, due to the reduced growth rates and to a shorter perpendicular wavelength; this result is plotted in figure 3(b) which also makes it apparent that the first stabilizing mechanism (on $\gamma$ ) dominates at high poloidal wave vectors. This conclusion is consistent with results previously obtained with the linear global code LORB [28, 29].

As a first qualitative survey of the effect of plasma shape on plasma confinement, one can perform a two dimensional scan in edge triangularity and edge elongation with a given pressure and current profile. The scan is performed by taking density and temperature profiles of a given pulse (28014 in our case) and then by changing the equilibrium reconstruction: i.e. recalculating the equilibrium starting with the same pressure and current profile and imposing a different shape to the LCFS. The recalculated $q$ profiles change up to about $10 \%$ along the maximum $\delta$ scan at each value of elongation. The maximum change in elongation at fixed triangularity is about $20 \%$, almost irrespectively of triangularity. The absolute difference stays almost constant up to the magnetic axis. 


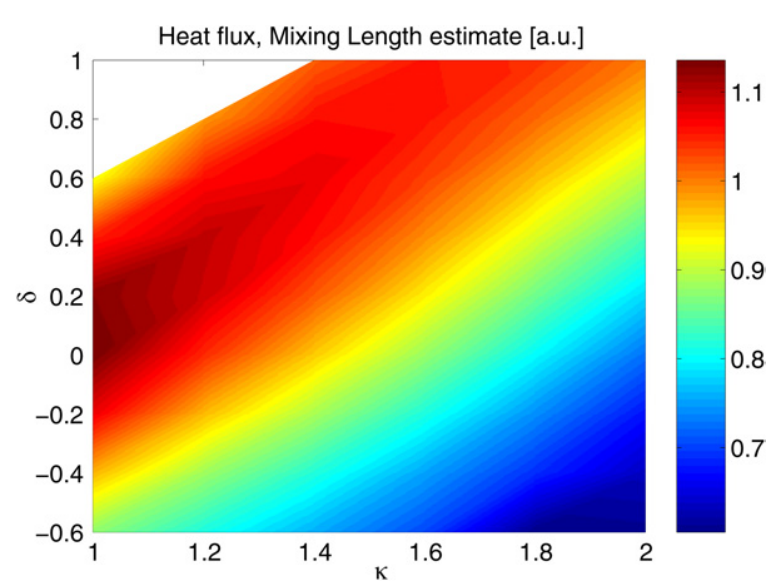

Figure 4. (Color online.) Mixing-length estimate, calculated as the maximum of $\gamma /\left\langle k_{\perp}^{2}\right\rangle$, of the electron heat flux obtained with GS2 at $\rho=0.7$ as a function of edge triangularity and elongation.

The resulting mixing-length heat diffusivity, calculated as the maximum of $\gamma /\left\langle k_{\perp}^{2}\right\rangle$ [27], as a function of the shape parameters is shown in figure 4. It is apparent that turbulence is quenched by lowering triangularity, especially to negative values, or by increasing elongation, as the contour levels over most of the $\kappa-\delta$ space can be approximately described by $\kappa \simeq \delta+$ const lines. A more detailed examination, however, reveals a more complex dependence on elongation at different values of triangularity. In fact, an increase in $\kappa$ is always stabilizing at negative $\delta$, whereas for positive triangularities it is initially destabilizing and then stabilizing again.

Next, we proceed to explore whether the variations induced by a change in triangularity in other discharge quantities could themselves have an influence on microturbulence. Since the TCV shots under analysis are dominated by TEM turbulence, one might speculate that the negative $\delta$ stabilization could be due to a difference in aspect ratio resulting in a different trapped particle fraction. In figure 5(a) we plot the inverse aspect ratio, calculated as

$$
\left(R_{\max }-R_{\min }\right) /\left(R_{\max }+R_{\min }\right)
$$

and, alternatively, in figure $5(b)$ through the expression

$$
\left\langle a / R_{0}\right\rangle=\frac{1}{R_{0}} \sqrt{\frac{\int_{\theta_{\min }}^{\theta_{\max }} \mathrm{d} \theta r^{2}|\tilde{\phi}(\theta)|^{2}}{\int_{\theta_{\min }}^{\theta_{\max }} \mathrm{d} \theta|\tilde{\phi}(\theta)|^{2}}},
$$

which, in analogy to equation (1), represents, by means of an average over the energy of the underlying instability, an effective aspect ratio seen by the particles (figure $5(b)$ ). Neither function shows the same trend as in figure 4, and the relative variation of the trapped particle fraction, which is proportional to the square root of the inverse aspect ratio, is a small fraction, less than $10 \%$, of the heat flux variation shown in figure 4.

Another effect that has to be accounted for is the dependence of local elongation on triangularity. Even with the same elongation of the LCFS, the difference in Shafranov shift for two different values of triangularity may result in a different degree of penetration of the elongation into the plasma core. This in turn could cause a variation in TEM turbulence in addition to the direct influence of triangularity. However, for TCV shots 28008 and 28014 the difference in elongation is less than $4 \%$ over the whole minor radius; this, considering figure 4 , 

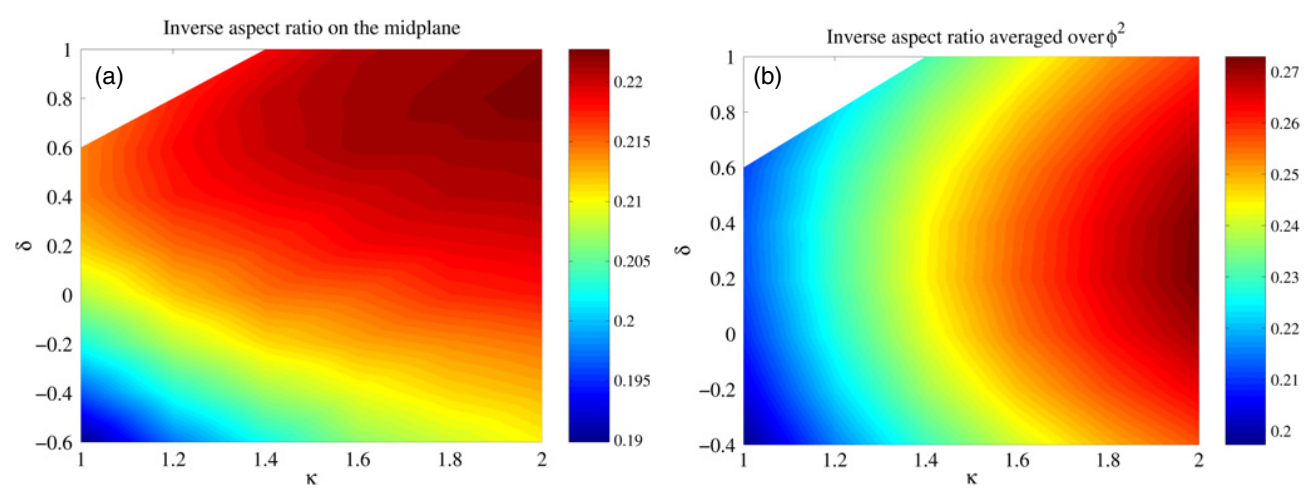

Figure 5. (Color online.) Inverse aspect ratio, defined by (a) equation (3) and (b) equation (4), as a function of triangularity and elongation of the LCFS.

can only have a negligible effect compared with the observed $30 \%$ reduction in the maximum of $\gamma /\left\langle k_{\perp}^{2}\right\rangle$.

Finally we consider the effect on the gradients induced by a change in triangularity. Indeed, even though the profiles as a function of the flux surface coordinate are identical, the local values of the spatial gradients generally differ due to flux surface compression. In particular, the enhanced Shafranov shift induced by negative triangularities compared with positive ones, leads to a more peaked pressure profile on the low field side, whereas the opposite happens on the high field side. To investigate this effect we performed a pressure gradient scan on linear simulations and compared the results expressed as a function of $(1 / T) \mathrm{d} T / \mathrm{d} \rho$ or $\langle\nabla \rho\rangle(1 / T) \mathrm{d} T / \mathrm{d} \rho$; if this effect was responsible for the difference under consideration the growth rates should be equal when plotted as a function of the latter quantity. Even though the difference in the growth rates almost halves, this effect cannot be responsible for the observed disparity.

The TEM was first theoretically investigated in [30], leading to the identification of the toroidal precession drift of trapped particles as the cause of the instability. Indeed, other parameters such as $T_{\mathrm{e}} / T_{\mathrm{i}}$ and density and temperature scale lengths being equal, which is the case in the present experiments, it is natural to search for the cause of the observed dependence in the drifts induced by the magnetic geometry. Even though the only operational difference between these TCV shots is the edge triangularity, this translates into differences in several quantities, both macroscopic, such as the Shafranov shift, and microscopic, such as the magnetic drifts. To understand how the different microscopic drifts interact, the positive triangularity case has been changed artificially by replacing one or more drives in the GK equation, one at a time, with their corresponding values taken from the negative triangularity case. In particular, in the toroidal GK equation one could isolate the effects of curvature and $\nabla B$ drifts (which have been simultaneously changed because they differ only in the negligible $\nabla \beta$ and are indicated as $\omega_{\mathrm{D}}$ in table 2), the parallel advection (indicated as $\nabla_{\|}$in table 2) and the gradient of the ballooning eikonal, which reflects the effect of magnetic shear and can be interpreted as $k_{\perp}$ (this parameter is indicated as $\nabla_{\perp}$ in table 2 and has been changed independently of the perpendicular drifts for the sake of numerical investigation). The result of this linear test is depicted in table 2, which reports the heat diffusivities, normalized to the positive triangularity case value, calculated as the maximum of $\gamma /\left\langle k_{\perp}^{2}\right\rangle$ [27]. In table 2 it is evident that parallel and perpendicular dynamics behave differently: the curvature and $\nabla B$ drifts together with $k_{\perp}$ act to reduce the linear $\chi_{\mathrm{ML}}$ of the perturbation in the negative $\delta$ 
Table 2. Normalized heat diffusivity through mixing-length estimate of real cases $(\delta= \pm 0.4$ at $\rho=0.7$ ) and of the artificially changed equilibria explained in the text (all the others).

\begin{tabular}{lllllllll}
\hline Equil. & $\delta=0.4$ & $\delta=-0.4$ & $\omega_{\mathrm{D}}$ & $\omega_{\mathrm{D}}+\nabla_{\perp}$ & $\omega_{\mathrm{D}}+\nabla_{\|}$ & $\nabla_{\perp}$ & $\nabla_{\perp}+\nabla_{\|}$ & $\nabla_{\|}$ \\
\hline$\chi_{\mathrm{ML}}$ & 1 & 0.77 & 0.90 & 0.74 & 0.92 & 0.82 & 0.84 & 0.98 \\
\hline
\end{tabular}

case, whereas the parallel advection does not appreciably influence it. In particular, the $\nabla_{\perp}$ of a negative $\delta$ configuration narrows the electrostatic potential which, in turn, reduces the equivalent perpendicular wavelength of the fluctuation.

\section{Non-linear simulations}

The linear simulations discussed in the previous section show a qualitative trend matching the one observed in the experimental TCV shots. Nevertheless it should be noted that linear mixing-length estimates only give a $30 \%$ difference in diffusivity between the two equilibria, whereas the experimental value is about $100 \%$ : non-linear effects might therefore be paramount in accounting for the larger variation (factor 2) observed in the experiment. Using the numerical grids described in section 2, non-linear runs have been performed to check whether the $E \times B$ non-linearity plays any role in the microstability analysis of these shots. As will be discussed in the following, it is indeed observed that non-linearity plays a further stabilizing role, increasing the difference between the positive and the negative triangularity cases, thus showing how the mixing length approximation is not adequate in describing all the physical details even if it succeeds in capturing the relevant trends. This can be appreciated in figure 6 , which compares the ratio between the experimental thermal diffusivities, reconstructed from a power balance analysis, of the two TCV pulses under analysis and the corresponding simulated ratios at three radial points. The non-linear simulations are performed in the collisionless limit. It can be seen that the reduction in transport with negative triangularity is qualitatively reproduced, but a satisfactory quantitative match is only obtained near the plasma edge. The smaller ratios seen in the simulation toward the inside of the plasma can be explained by the finite penetration depth of triangularity. In particular, if at $\rho=1$ the triangularities are \pm 0.4 , at $\rho=0.7$ they are equal to \pm 0.17 and at $\rho=0.4$ they are lower by a further factor of 3 , being equal to about \pm 0.07 (see figure 1). So since, going toward the magnetic axis, the fraction of trapped particles tends to zero and the difference in triangularity vanishes, the resulting difference in the trapped particle contribution to the total transport is annulled. Even though passing particles are also stabilized by negative triangularity, they contribute less to the total transport and with a smaller absolute difference with respect to the trapped particles. All this results in the two simulations giving approximately the same result. This claim is supported by figure 7, which shows the decrease in the trapped particle contribution to the total transport for the two extreme radial locations in figure 6.

Since the density and temperature profiles are everywhere equal in the two shots, the anomalous transport reduction has to occur over the whole minor radius; the doubling of the experimental confinement time can therefore not be explained by a stiffness argument which would imply an increased stored energy due to a stabilization more or less localized close to the plasma edge. In particular, the experimental diffusivity ratio is still approximately 2 even at the innermost location studied. Since a local analysis is patently unable to reproduce this result, as discussed above, global effects may be at play which cannot be reproduced by the present modeling. In the following all the numerical analyses will be performed at $\rho=0.7$. 


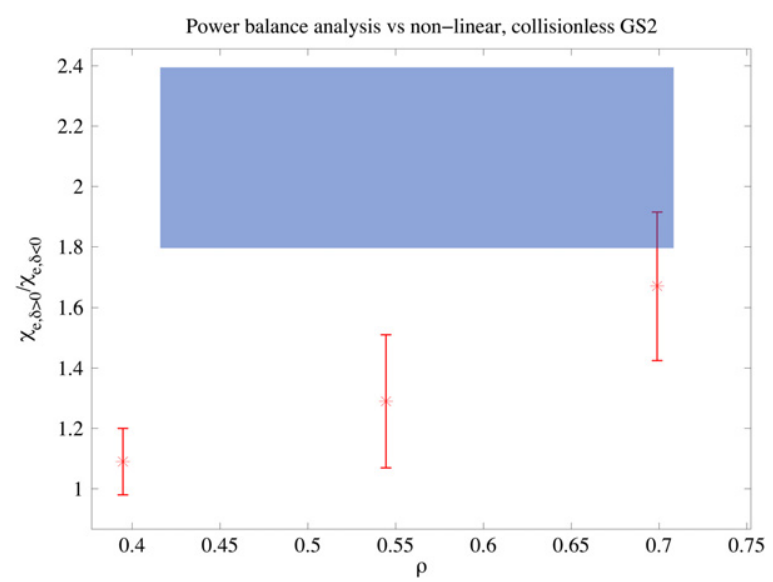

Figure 6. (Color online.) Horizontal rectangle: range in which lies the ratio of experimental electron thermal conductivities, as a function of the square root of the normalized volume, between discharges with edge triangularities equal to \pm 0.4 . The area has been stopped where the EC power was deposited $(\rho<0.4)$ and where the impurity ray emission is too large, thus spoiling the measurement $(\rho>0.7)$. Points: same ratio simulated by GS2; the mean values are calculated in the saturated phase of the simulation.
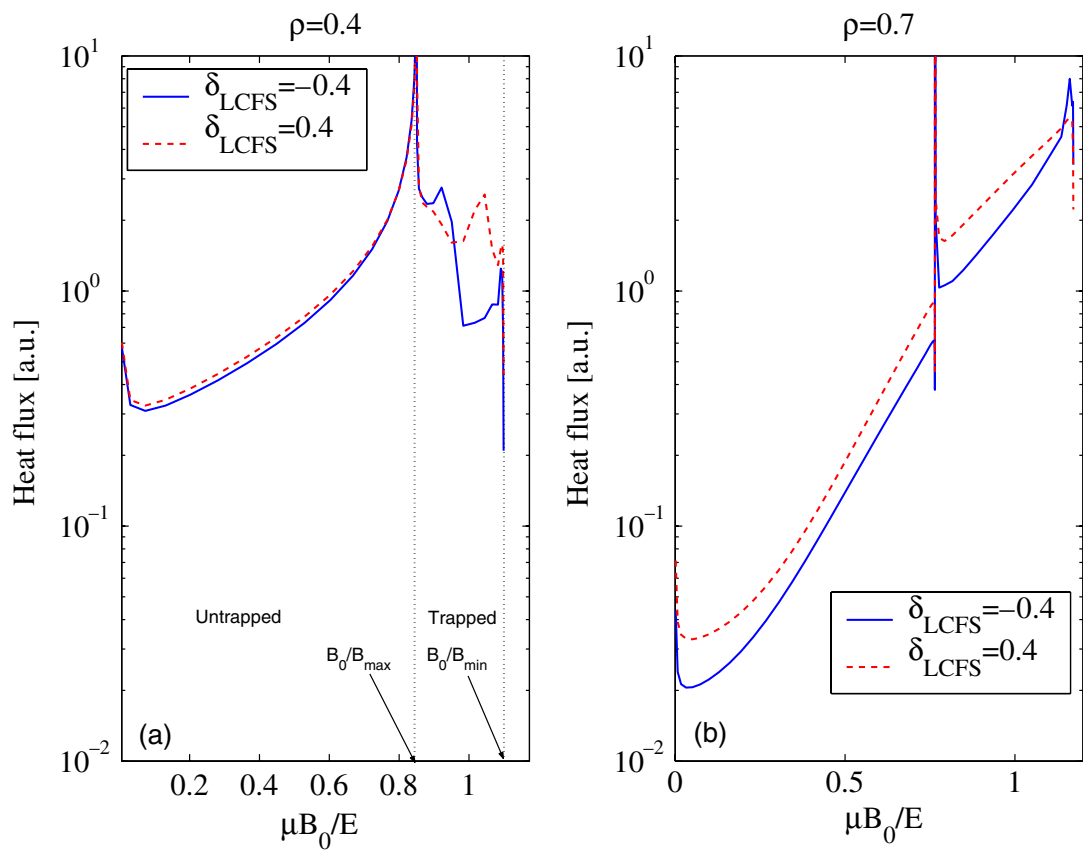

Figure 7. (Color online.) Comparison of the heat transport as a function of pitch angle for positive and negative triangularities calculated at $\rho=0.4(a)$ and $0.7(b)$.

Experimentally, the electron diffusivity is found to depend on a combination of various physical quantities such as $T_{\mathrm{e}}, n_{\mathrm{e}}$ and $Z_{\text {eff }}$. In particular, an inverse linear scaling was found with respect to a particular combination corresponding to the effective collisionality, i.e. the electron collisionality normalized to the electron drift frequency, with the proportionality factor 


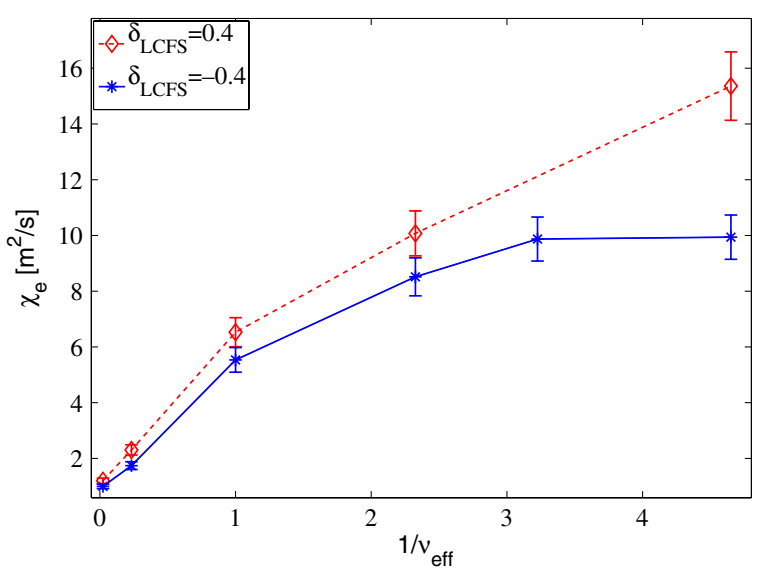

Figure 8. Effect of collisionality on the non-linearly simulated electron heat diffusivity.

depending in turn on triangularity [1]. As can be seen in figure 8, non-linear collisional simulations reproduce the same behavior with numerical values of the same order of magnitude as the experimental ones. In these non-linear simulations the estimated saturated heat flux is carried primarily by the electron species and, additionally, the electron heat flux is mainly due to trapped particles (see figure 7), which confirms the TEM nature of the turbulence under investigation. This insight works in favor of an intuitive explanation of the collisionality dependence observed in both simulations and experiments. In fact collisional detrapping processes alter the phase space configuration, leading to more and more particles being taken out of the highly unstable trapped region and transferred into the less unstable passing region, where they therefore contribute less to the overall transport. This is also reflected in a higher relative contribution of the passing electrons to the total (and decreased) calculated heat flux increasing, for the cases plotted in figure 9 , from 5 to $10 \%$. Thus, in terms of the effect on heat transport, reversing the triangularity from positive to negative is equivalent to increasing the collisionality by a given factor. In other words, TEMs in a negative triangularity plasma are stabilized as if the plasma was much more collisional; thus allowing the same amount of transport with, for example, a much lower density and/or a much cleaner plasma. In figure 9 the particle contribution to the total transport as a function of pitch angle is given by the slope of the curves, so it is possible to appreciate how the roles of barely trapped and barely passing electrons become increasingly similar as collisionality is increased.

\section{Investigation of instability drives}

Artificial changes in the equilibrium can be applied to non-linear simulations in a similar manner as was discussed in section 3 for the linear case. The conclusions of that section concerning the roles of the individual drives can thus be put to the test in the non-linear case. In table 3 it can be seen how a change in either the $\nabla B$ and curvature drift or in the equivalent $k_{\perp}$ entering in the quasi-neutrality calculations and gyroaveraging, lead to an approximately equal degree of turbulence suppression. Thus the slight difference found between these two terms in the linear approximation essentially disappears in the non-linear phase. However it is important to note that when these terms are changed simultaneously, which has to be the case in order for the simulations to be physically meaningful since $\omega_{\mathrm{D}}=\vec{\nabla} S \cdot \vec{B}_{0} \wedge\left(m v_{\|}^{2} \hat{b}_{0} \cdot \vec{\nabla} \hat{b}_{0}+\mu \vec{\nabla} B_{0}+Z_{\mathrm{e}} \vec{\nabla} \Phi_{0}\right) /\left(m B_{0} \Omega\right)$ [26], the heat flux variation is 

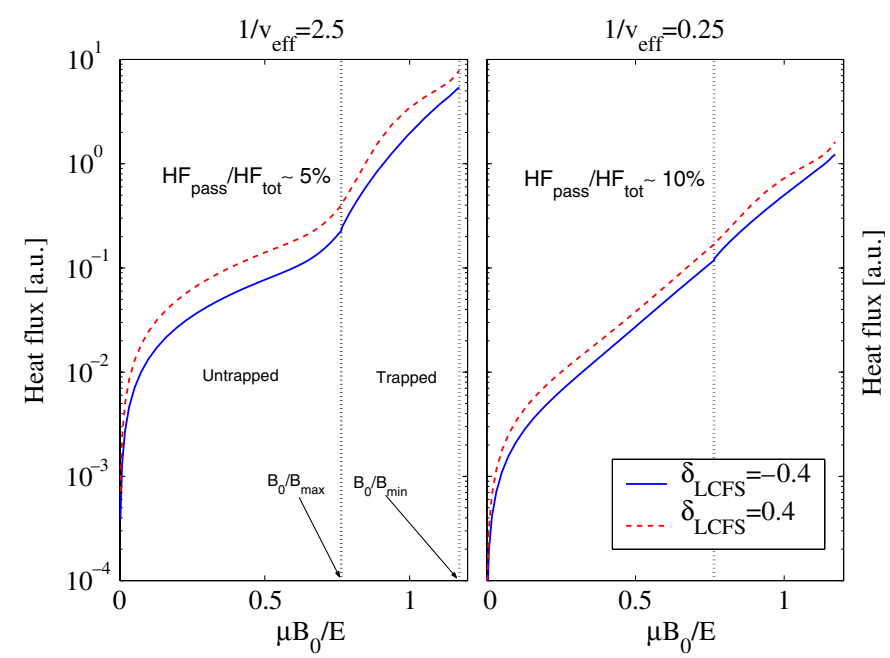

Figure 9. Heat flux integrated over pitch angle (cumulative integral) versus pitch angle for two values of collisionality. At higher $v_{\text {eff }}$ the relative contribution from passing electrons to the total flux increases. Even though their contribution to the total flux is small, note how negative $\delta$ also stabilizes passing electrons.

Table 3. Same as table 2 for corresponding non-linear simulations.

\begin{tabular}{ll}
\hline Case & Saturated heat flux \\
\hline$\delta>0$ & 1 \\
$\delta<0$ & 0.59 \\
$\omega_{\mathrm{D}}$ & 0.55 \\
$\nabla_{\perp}$ & 0.53 \\
$\nabla \rho$ & 0.78 \\
$\nabla_{\perp}+\omega_{\mathrm{D}}$ & 0.58 \\
\hline
\end{tabular}

reproduced almost exactly, thus confirming that the observed stabilization is induced by a combined effect of different perpendicular drifts and consistent perpendicular scales of the fluctuations. In the table it is also evident how the $\nabla \rho$ term, which enters in the calculations of volume integrals and surface averages, is not alone responsible for the difference, which is then due to the linear terms, although the full non-linear equation increases the difference between the two triangularities compared with the results of table 2 .

Since, as already mentioned in section 3, TEMs are destabilized by the resonance between the real frequency of the perturbation and the toroidal precessional drift frequency of trapped electrons, it is interesting to evaluate the dependence of the toroidal precessional drift on triangularity. When the banana width can be neglected with respect to the equilibrium scale lengths, which is one of the assumptions of a local code, the toroidal precessional drift reads [31]

$$
\langle\dot{\varphi}\rangle=\frac{1}{e} \frac{\partial I_{\|} / \partial \psi}{\partial I_{\|} / \partial E},
$$

where $E$ is the energy of the particle, $e$ its charge and $I_{\|}$is the second (or longitudinal) adiabatic invariant, defined as

$$
I_{\|}(\psi, E, \mu)=\oint \mathrm{d} l_{\|} v_{\|}=2 \int_{-\theta_{\mathrm{b}}}^{\theta_{\mathrm{b}}} \mathrm{d} \theta J B\left|v_{\|}\right|
$$




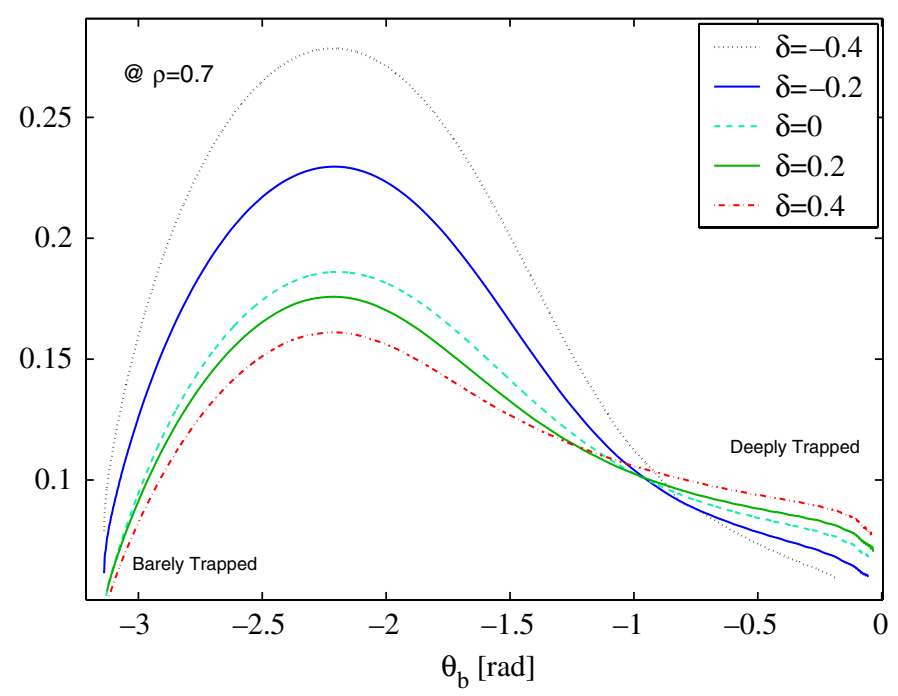

Figure 10. (Color online.) Toroidal precessional drift of a thermal trapped electron, in arbitrary units, as a function of the trapped particle bouncing points, for five different equilibria which have been generated from a single TCV shot, by changing the shape of the LCFS.

Here $\pm \theta_{\mathrm{b}}$ are the bouncing points of the electron with energy $E$ and pitch angle $\mu\left(B\left(\psi, \theta_{\mathrm{b}}\right)=\right.$ $E / \mu), J$ is the Jacobian of the transformation to the field-aligned coordinate system and $B$ is the amplitude of the magnetic field. Equation (5) can then be rewritten as

$$
\begin{aligned}
& \langle\dot{\varphi}\rangle(\psi, E, \mu)=\frac{1}{e} \frac{\partial I_{\|} / \partial \psi}{\partial I_{\|} / \partial E} \\
& \quad=\frac{2 E}{e} \frac{\partial}{\partial \psi}\left(\int_{-\theta_{\mathrm{b}}}^{\theta_{\mathrm{b}}} \mathrm{d} \theta J(\theta) B(\theta) \sqrt{1-B(\theta) / B\left(\theta_{\mathrm{b}}\right)}\right) / \int_{-\theta_{\mathrm{b}}}^{\theta_{\mathrm{b}}} \mathrm{d} \theta J(\theta) B(\theta) / \sqrt{1-B(\theta) / B\left(\theta_{\mathrm{b}}\right)},
\end{aligned}
$$

which is then a linear function of the particle energy.

Figure 10 shows that this quantity behaves very differently for deeply trapped and barely trapped particles, in particular the toroidal precessional drift tends to be higher for positive $\delta$ in the deeply trapped region while the opposite happens for negative $\delta$. For a geometrical interpretation we show, in figure 11, the shape of three equilibria and the bouncing angle represented in figure 10. In order to investigate the resonance condition between the perturbation and the toroidal precessional drift frequency, we normalize the latter to the real frequency of the linear mode which contributes most to the total transport, in agreement with the mixing-length estimate used in section 3. Figure 12 shows the ratio $n_{0}\langle\dot{\varphi}\rangle / \omega\left(n_{0}\right)$ for TCV shots 28008 and 28014, calculated for a particle energy equal to the thermal electron temperature.

This ratio is a linear function of the electron energy divided by the electron temperature and so, as a rule of thumb, the resonance condition is to be better evaluated at the energy where most of the transport occurs. Our GS2 simulations indicate that this happens for $2.5 \simeq E / T_{\mathrm{e}} \simeq 3$, which would push the resonance to be closer to the positive $\delta$ case in the whole pitch angle space but in the deeply trapped region. In order to verify this rough estimate we then calculate the heat flux by performing the energy integral. In this paper we showed how these experimental shots are largely dominated by TEM turbulence and, further, that there is no appreciable shape 


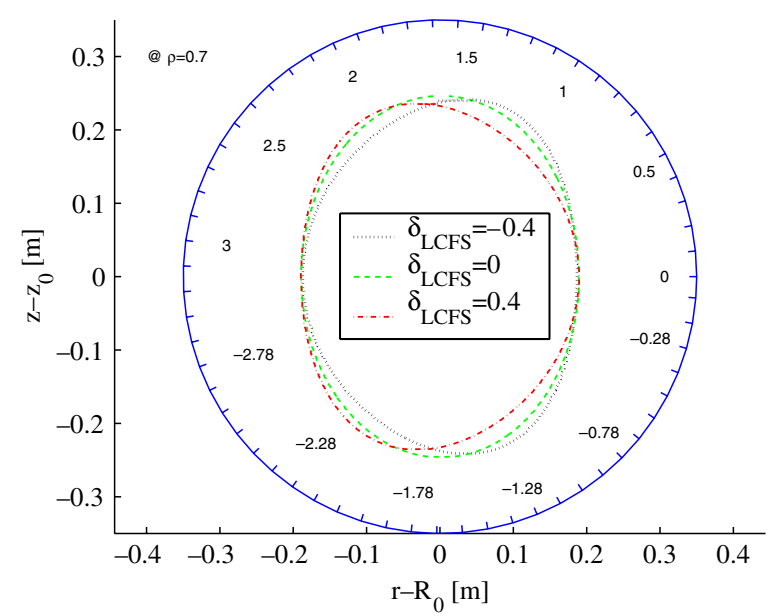

Figure 11. (Color online.) Shape of three equilibria of figure 10 superimposed to the bouncing angle, indicating where the toroidal precessional drift of the equilibria cross.

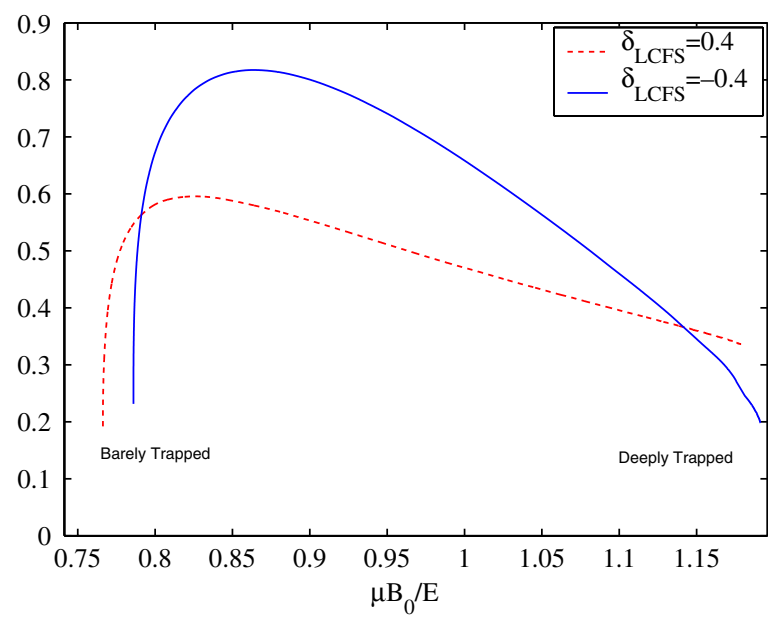

Figure 12. (Color online.) Toroidal precessional drift of a thermal trapped electron normalized to the real frequency of most linearly unstable mode as a function of the pitch angle.

effect on the parallel advection term in the GK equation; this now justifies the use of the bounce-averaged GK linear equation. Considering a generic physical quantity $X$, its flux across a given flux surface is given by

$\left\langle\Gamma_{X}\right\rangle=\frac{\int \mathrm{d}^{3} r \mathrm{~d} E \mathrm{~d} \mu X \tilde{g} v_{E \wedge B}^{\vec{\nabla}} \cdot \vec{\nabla} \rho}{\int \mathrm{d}^{3} r \nabla \rho}=-\frac{\int \mathrm{d}^{3} r \mathrm{~d} E \mathrm{~d} \mu X \tilde{g} \vec{\nabla} \phi \wedge \vec{b} / B \cdot \vec{\nabla} \rho}{\int \mathrm{d}^{3} r \nabla \rho}$,

where $\tilde{g}$ is the non-adiabatic part of the distribution function, $B$ the amplitude of the magnetic field and $\tilde{\phi}$ the electrostatic potential, and the integral is carried out over the whole flux tube. By expressing $\tilde{g}$ and $\tilde{\phi}$ as Fourier sums, equation (8) becomes

$$
\left\langle\Gamma_{X}\right\rangle=\frac{\int \mathrm{d}^{3} r \mathrm{~d} E \mathrm{~d} \mu X \Sigma_{k} \hat{g}_{k} \hat{\phi}_{k}^{*} \mathrm{i} \vec{k} \wedge \vec{b} / B \cdot \vec{\nabla} \rho}{\int \mathrm{d}^{3} r \nabla \rho} .
$$



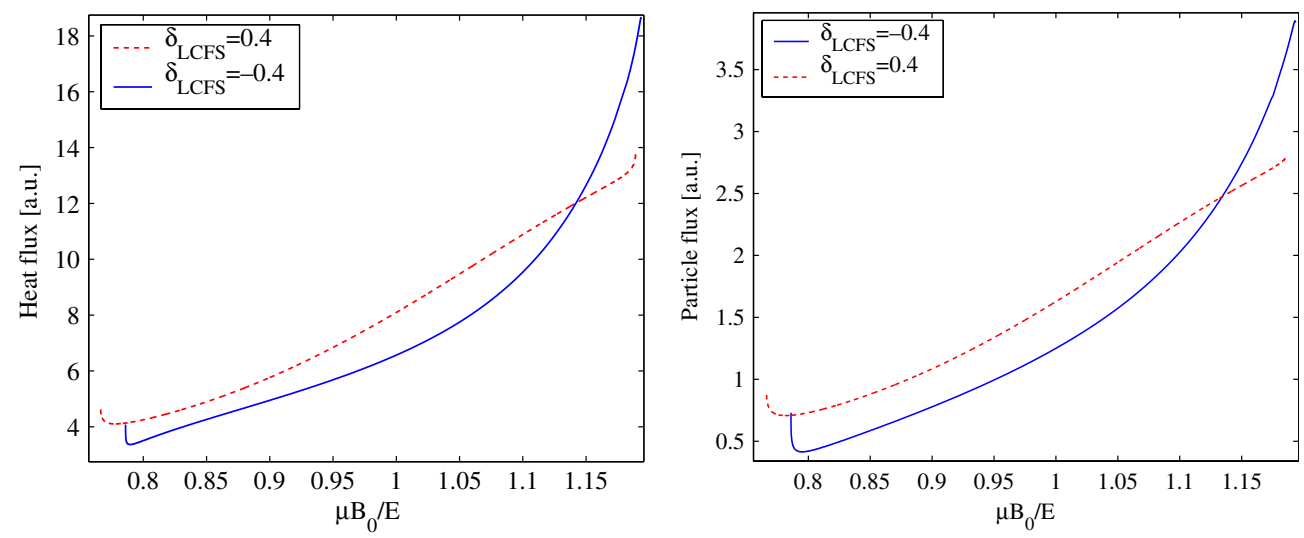

Figure 13. (Color online.) Linear estimate of the $X$ flux as a function of the pitch angle, for TCV shots 28008 and 28014.

Since in the linear theory there is no saturation mechanism, let us normalize the $X$ flux to the squared modulus of the electrostatic potential. Considering only the mode which contributes most to the total transport we may then write

$$
\left\langle\Gamma_{X}\right\rangle=\mathfrak{R} \frac{\int \mathrm{d}^{3} r \mathrm{~d} E \mathrm{~d} \mu X \hat{g}_{k} \hat{\phi}_{k}^{*} \mathrm{i} \vec{k} \wedge \vec{b} / B \cdot \vec{\nabla} \rho}{\frac{e^{2}}{T_{\mathrm{e}}^{2}} \int \mathrm{d}^{3} r \hat{\phi}_{k} \hat{\phi}_{k}^{*} \int \mathrm{d}^{3} r \nabla \rho} .
$$

The bounce-averaged kinetic equation in Fourier space reads [32]

$$
\tilde{g}=\frac{\omega-\omega^{*}\left[1-\eta_{\mathrm{e}}\left(\frac{3}{2}-\frac{E}{T_{\mathrm{e}}}\right)\right]}{\omega-n_{0}\langle\dot{\varphi}\rangle} \frac{e\langle\phi\rangle}{T_{\mathrm{e}}} \mathrm{e}^{-E / T_{\mathrm{e}}},
$$

where $\eta_{\mathrm{e}}=\mathrm{d} \log \left(T_{\mathrm{e}}\right) / \mathrm{d} \log \left(n_{\mathrm{e}}\right),\langle\phi\rangle$ is the bounce-averaged electrostatic potential, $\langle\dot{\varphi}\rangle$ is the bounce-averaged toroidal precessional drift frequency, $\omega$ is the mode's complex frequency and the diamagnetic frequency is expressed by $\omega^{*}=k_{\theta} T_{\mathrm{e}} \nabla n_{\mathrm{e}} /\left(q B n_{\mathrm{e}}\right)$. Adopting the approximation $\phi \simeq\langle\phi\rangle$ and substituting equation (11) into equation (10) we obtain the following quasi-linear estimate for the $X$ flux

$$
\left\langle\Gamma_{X}\right\rangle=\Re \int \mathrm{d}^{3} r \mathrm{~d} E \mathrm{~d} \mu X \frac{\omega-\omega^{*}\left[1-\eta_{\mathrm{e}}\left(\frac{3}{2}-\frac{E}{T_{\mathrm{e}}}\right)\right] \mathrm{i} \vec{k} \wedge \vec{b} / B \cdot \vec{\nabla} \rho}{\left(\omega-n_{0}\langle\dot{\varphi}\rangle\right)} \mathrm{e}^{-E / T_{\mathrm{e}}} / \frac{e}{T_{\mathrm{e}}} \int \mathrm{d}^{3} r \nabla \rho .
$$

Taking now as $X$ the particle energy, equation (12) gives the heat flux, while taking $X=1$ we obtain the particle flux.

Figure 13 shows the $\mu$ integrand (i.e. energy and surface integral) of equation (12) for TCV shots 28008 and 28014, indicating how negative triangularity acts, compared with positive triangularity, favorably everywhere in the pitch angle space except for the deeply trapped particles, which are less unstable in a positive triangularity configuration. Figure 11 indicates that this region is a subset of the bad curvature region. Roughly the same result is reproduced qualitatively also by linear and non-linear GS2 simulations (figure 14) which solve the whole GK equation coupled to the Poisson equation. The only difference in the non-linear run is the actual ratio of the two heat fluxes and the pitch angle value at which they cross over each other.

As the toroidal precessional drift seems to be playing such an important role in the microinstability of these TCV shots, it could be expected that its radial dependence would be a good diagnostic for interpreting the results of GS2. In figure 15 we plot, as a function of 

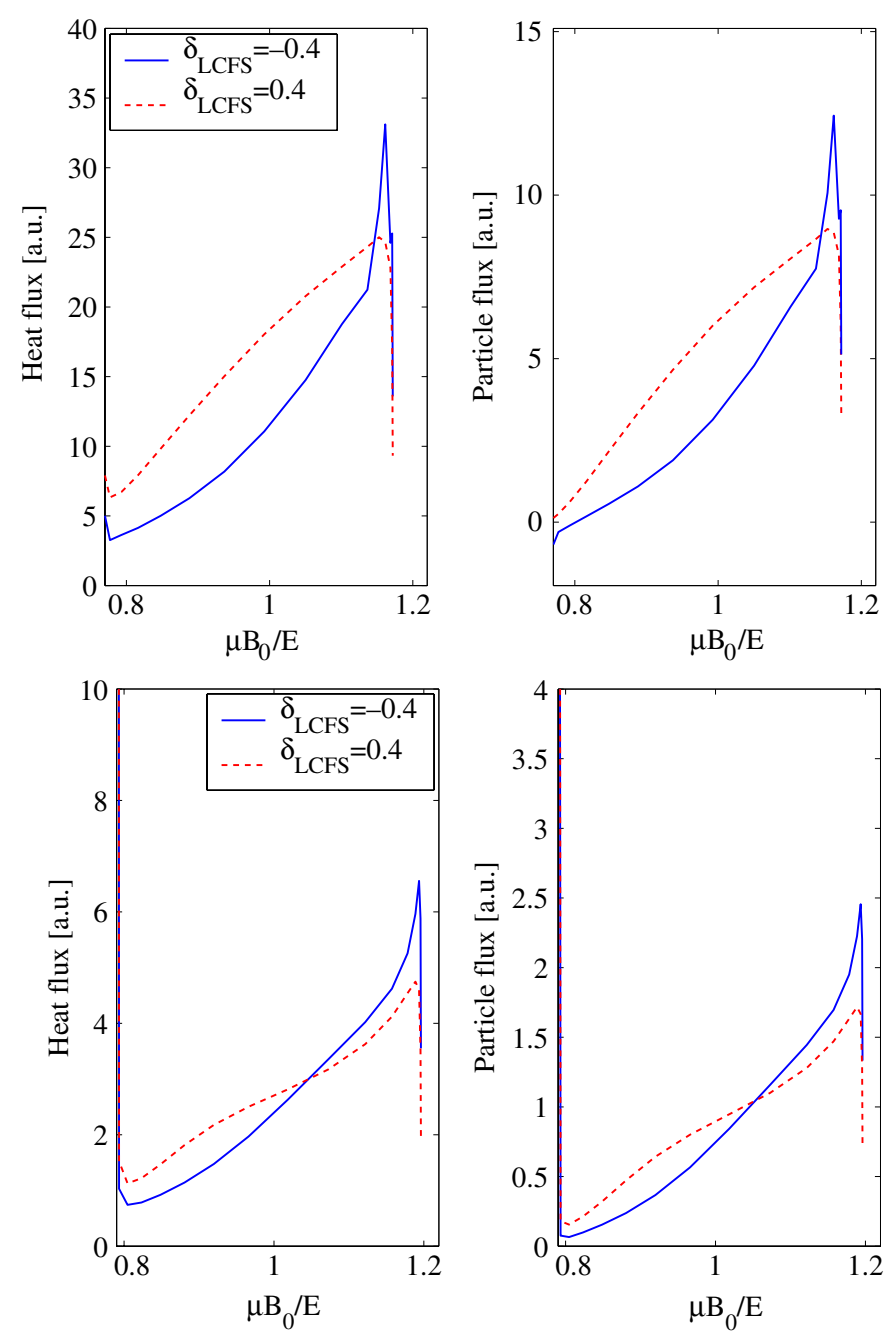

Figure 14. (Color online.) Non linear (top) and linear (bottom) GS2 estimates of the heat flux (left) and of the particle flux (right) as a function of the pitch angle, for TCV shots 28008 and 28014.

the trapped particle bounce-angle, the value of $\langle\dot{\varphi}\rangle$ for TCV shots 28008 and 28014 at three radial positions corresponding to the ones in figure 6 . The difference between the two cases gets smaller and smaller as one approaches the magnetic axis, due to the finite penetration depth of triangularity.

As briefly mentioned in section 3, since the toroidal precessional drift is a combined effect of parallel and perpendicular dynamics, the $\langle\dot{\varphi}\rangle$ diagnostic is not only consistent with the numerical exercises of tables 2 and 3 , but is actually a direct and powerful tool to interpret in detail the transport properties of plasmas dominated by TEM turbulence.

\section{Conclusions}

In this paper a quantitative comparison of TCV shots with linear and non-linear local GK modeling has been performed to investigate the effect of plasma triangularity. 

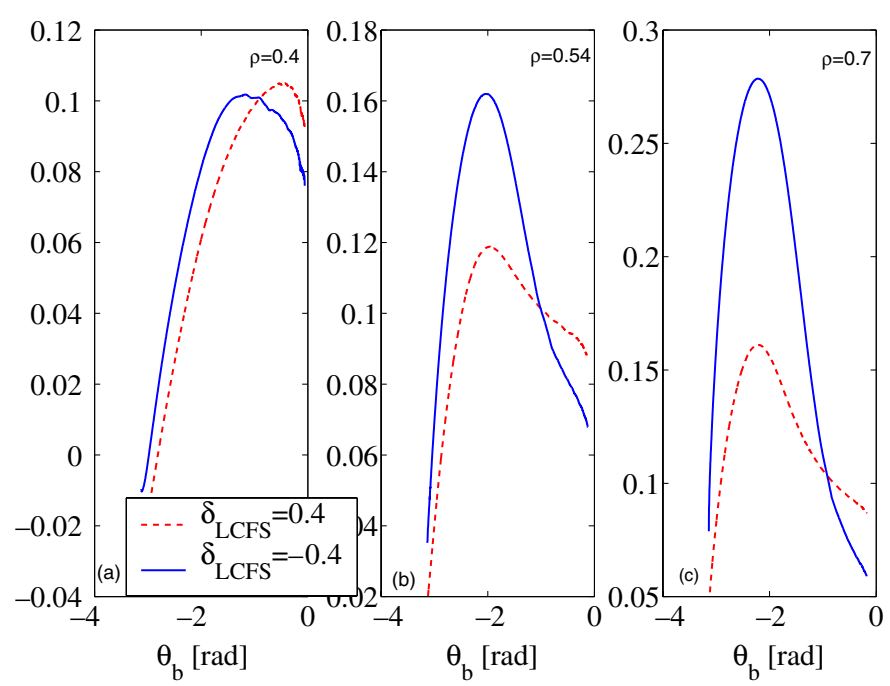

Figure 15. (Color online.) Toroidal precessional drift frequency, in arbitrary units, as a function of the bouncing angles [rad] of a trapped particle with energy equal to the plasma electron temperature, calculated at three radial positions.

Experimentally a negative triangularity was found to reduce electron heat transport in EC-heated L-mode TCV discharges, to the point of doubling the electron confinement time when reversing the edge triangularity from $\delta_{\mathrm{LCFS}}=0.4$ to $\delta_{\mathrm{LCFS}}=-0.4$ [1].

The TCV shots under consideration are found to be dominated by TEM, which would thus need to be considered partially stabilized by negative triangularities.

Both linear and non-linear simulations reproduce the experimental trend qualitatively, and the non-linear analysis in particular also provides a satisfactory quantitative agreement. This, however, is only true close to the plasma edge, due to the finite penetration depth of triangularity. Since on the contrary the experimental stabilization is almost uniform across the minor radius, the present modeling cannot explain all the physical effects at play, in particular those that could arise from global mechanisms.

Experimentally, the heat transport was found to be reduced by plasma collisionality and, indeed, this has been reproduced by the simulations. This is in agreement with the intuitive picture of collisional detrapping processes altering the phase space configuration and leading to more and more particles being taken out of the highly unstable trapped region and transferred into the less unstable passing region, where they therefore contribute less to the overall electron transport.

It has been shown by linear and non-linear numerical analyses that the favorable effect played by negative triangularity is dominated by the perpendicular drifts and by the effective $k_{\perp}$. In particular, the effective stabilization is a result of a complex interplay of deeply and barely trapped particles which react in opposite ways to triangularity. This, in turn, is due to the electron toroidal precessional drift, which is larger with $\delta$ in the deeply trapped spectral region, while the opposite is true elsewhere in the pitch angle space.

\section{Acknowledgments}

The authors would like to thank Paolo Ricci for useful discussions, Trach-Minh Tran and Alberto Bottino for valuable assistance and the authors of the GS2 code for releasing the 
source. Simulations were performed on the Linux clusters PLEIADES and PLEIADES2 of the Ecole Polytechnique Fédérale de Lausanne. This work was supported in part by the Swiss National Science Foundation.

\section{Appendix. Geometrical background}

In this section we briefly elucidate some geometrical concepts useful to the reader not familiar with the GS2 code and, in general, with ballooning coordinates. An axisymmetric equilibrium magnetic field made of closed surfaces may be represented in terms of scalar potentials [25]

$$
\vec{B}=\vec{\nabla} \alpha \wedge \vec{\nabla} \psi, \quad \alpha=\varphi-q(\psi) \theta-v(\psi, \theta),
$$

where $q$ is the safety factor and the scalar potentials are $\psi$, the poloidal flux, $\theta$, the poloidal angle, and $\varphi-v$, the toroidal angle minus $\nu$, which is a periodic function in $\varphi$ and $\theta$. The field lines are straight and lie in the $(\varphi-\nu, \theta)$ plane. A convenient coordinate system is $(\psi, \alpha, \theta)$, where $\psi$ defines the flux surface of interest, $\alpha$ identifies each field line on a flux surface and $\theta$ labels the position along the field line $\alpha$ on the flux surface $\psi$.

In ballooning coordinates, the quasi-orthogonality between the equilibrium magnetic field, $\vec{B}_{0}$, and the dominant instability is modeled by assuming that any perturbation of a given quantity $X$ may be expressed as [26]

$$
X=\hat{X}(\theta) \mathrm{e}^{\mathrm{i} S},
$$

where $\hat{X}$ is the complex amplitude of the perturbation and $S$ is the ballooning eikonal, such that

$$
\vec{B}_{0} \cdot \vec{\nabla} S=\vec{\nabla} \alpha \wedge \vec{\nabla} \psi \cdot \vec{\nabla} S=0 .
$$

This condition models the alignment of perturbations along the field line. In turn this implies that $S$ must be a function of $\psi$ and $\alpha$, and could thus be expressed by $S=n_{0}\left[\alpha+q(\psi) \theta_{0}\right]$. Here $n_{0}$ is an integer labeling the mode instability and $\theta_{0}$ is the ballooning angle which ties radial and poloidal mode numbers through the relation $k_{x}=-k_{\theta} s \theta_{0}$, where $s$ is the magnetic shear [26].

The non-linear GK equation, in ballooning coordinates, is given by

$$
\left(\frac{\partial}{\partial t}+v_{\|} \vec{b} \cdot \vec{\nabla}+[\tilde{\chi}, \cdot] / B+\mathrm{i} \omega_{\mathrm{D}}\right) \tilde{g}=\mathrm{i} \omega_{*}^{T} \tilde{\chi}-e \frac{\partial F_{0}}{\partial E} \frac{\partial \tilde{\chi}}{\partial t},
$$

where $\tilde{g}$ is the non-adiabatic part of the distribution function, $F_{0}$ is the Maxwellian equilibrium distribution function, $E$ the energy coordinate, $[\cdot, \cdot]$ is the Poisson bracket of the spatial derivatives which models the non-linear $E \times B$ drift, $\omega_{\mathrm{D}}=\vec{\nabla} S \cdot \overrightarrow{B_{0}} \wedge\left(m v_{\|}^{2} \hat{b}_{0} \cdot \vec{\nabla} \hat{b}_{0}+\mu \nabla B_{0}+\right.$ $\left.Z_{\mathrm{e}} \vec{\nabla} \Phi_{0}\right) /\left(m B_{0} \Omega\right)$ the curvature and $\nabla B$ drift, $\tilde{\chi}=J_{0}\left(k_{\perp} \rho_{\mathrm{i}}\right) \tilde{\phi}$ the gyro-averaged electrostatic potential and $\omega_{*}^{T}=\omega_{*} \partial F_{0} / \partial \psi$, where $\omega_{*}$ is the diamagnetic frequency. In this equation only the parallel advection $\vec{b} \cdot \vec{\nabla}$, the $k_{\perp}$ term, the curvature and $\nabla B$ drift operators and the magnetic field itself depend on the coordinate $\theta$ along the field line [26], and so may change according to the equilibrium reconstruction. Also an additional term, $\nabla \rho$, depends on $\theta$ and enters in the evaluation of volume integrals and flux surface averages.

Once the toroidal mode number $n_{0}$ of the perturbation and the radial derivative of the equilibrium distribution function are fixed, any change in the equilibrium reconstruction is reflected into changes in the afore-mentioned quantities.

Let us now develop one of these terms, $\left|k_{\perp}\right|^{2}$, which has been used in the paper

$\left|k_{\perp}\right|^{2}=|\vec{\nabla} S|^{2}=n_{0}^{2}\left|\vec{\nabla}\left(\alpha+q \theta_{0}\right)\right|^{2} \propto k_{\theta}^{2}\left|\vec{\nabla} \alpha \cdot \vec{\nabla} \alpha+2 \theta_{0} \vec{\nabla} \alpha \cdot \vec{\nabla} q+\theta_{0}^{2} \vec{\nabla} q \cdot \vec{\nabla} q\right|$.

In the circular, high aspect ratio, zero $\beta$, limit this can be written as

$$
|\vec{\nabla} S|^{2} \propto k_{\theta}^{2}\left|1+s^{2} \theta^{2}-2 s^{2} \theta_{0} \theta+\theta_{0}^{2} s^{2}\right| .
$$




\section{References}

[1] Camenen Y et al 2007 Nucl. Fusion 47510

[2] Turnbull A D et al 1988 Nucl. Fusion 281379

[3] Hofmann F et al 1998 Phys. Rev. Lett. 812918

[4] Lazarus E A et al 1991 Phys. Fluids B 32220

[5] Ferron J R et al 2005 Phys. Plasmas 12056125

[6] Ongena J et al 2001 Plasma Phys. Control. Fusion 43 A11

[7] Troyon F et al 1984 Plasma Phys. Control. Fusion 26209

[8] Hua D D et al 1992 Phys. Fluids B 43216

[9] Waltz R E and Miller R L 1999 Phys. Plasmas 64265

[10] Rewoldt G et al 1982 Phys. Fluids 25480

[11] Kotschenreuther M, Rewoldt G and Tang W M 1995 Comput. Phys. Commun. 88128

[12] Belli E et al 2008 Phys. Plasmas 15092303

[13] Jolliet S et al 2007 Comput. Phys. Commun. 177409

[14] Angelino P et al 2006 Plasma Phys. Control. Fusion 48557

[15] Kinsey J E et al 2007 Phys. Plasmas 14102306

[16] Hofmann F et al 1986 Fusion Technol. 1687

[17] Moret J-M et al 1997 Phys. Rev. Lett. 792057

[18] Weisen H et al 1997 Nucl. Fusion 371741

[19] Hofmann F et al 2001 Plasma Phys. Control. Fusion 43 A161

[20] Pochelon A et al 1999 Nucl. Fusion 391807

[21] Camenen Y et al 2005 Plasma Phys. Control. Fusion 471971

[22] Boucher D and Rebut P H 1992 Proc. IAEA Technical Conf. on Advances in Simulation and Modeling in Thermonuclear Plasmas (Montreal, QC, Canada) 142

[23] Lütjens H, Bondeson A and Sauter O 1996 Comput. Phys. Commun. 97219

[24] Miller R L et al 1998 Phys. Plasmas 5973

[25] Greene J M and Johnson J L 1962 Phys. Fluids 5510

[26] Antonsen T M Jr and Lane B 1980 Phys. Fluids 231205

[27] Jenko F et al 2005 Plasma Phys. Control. Fusion 47 B195

[28] Bottino A et al 2004 Phys. Plasmas 11198

[29] Camenen Y PhD Thesis EPFL, LRP 821/06

[30] Kadomtsev B B and Pogutse O P 1966 Zh. Eksp. Teor. Fiz. 511734 Kadomtsev B B and Pogutse O P 1967 Sov. Phys._JETP 241172

[31] Rosenbluth M and Sloan M L 1971 Phys. Fluids 141725

[32] Gang F Y and Diamond P H 1990 Phys. Fluids B 22976 